FACTA UNIVERSITATIS (NIŠ)

Ser. Math. Inform. Vol. 36, No 2 (2021), 249-257

https://doi.org/10.22190/FUMI200428019C

Original Scientific Paper

\title{
NUMERICAL SOLUTIONS OF NONLINEAR FRACTIONAL DIFFERENTIAL EQUATIONS VIA LAPLACE TRANSFORM
}

\author{
Süleyman Çetinkaya ${ }^{1}$ and Ali Demir ${ }^{2}$ \\ ${ }^{1}$ Faculty of Arts and Sciences, Department of Mathematics, \\ P. O. Box 60, 41380 Kocaeli, Turkey \\ 2 Faculty of Arts and Sciences, Department of Mathematics, \\ P.O. Box 73, 41380 Kocaeli, Turkey
}

\begin{abstract}
In this study, solutions of time-space fractional partial differential equations (FPDEs) are obtained by utilizing the Laplace transform iterative method. The utility of the technique is shown by getting numerical solutions of various FPDEs.

Key words: differential equations, Laplace transform, Fractional derivatives and integrals, Functional-differential equations
\end{abstract}

\section{Introduction}

Mathematical models by fractional differential equations for various physical phenomena play important roles in all applied sciences such as mathematics physics, biology, dynamical systems, control systems, engineering and so on $[1,2,3,4,5,6,7$, $8,9,10,11,12]$. Also, there are various studies on fractional diffusion equations. Exact analytical solutions of heat equations are obtained by using operational method [13]. The existence, uniqueness and regularity of solution of impulsive sub-diffusion equation are established by means of eigenfunction expansion [14]. The anomalous diffusion models with non-singular power-law kernel have been investigated and constructed [15]. Moreover, nonlinear fractional partial differential equations (FPDEs) are employed in modeling various nonlinear phenomena, mainly dealing with memory, and they present a crucial role in technology and science. Taking

Received April 28, 2020; accepted: December 11, 2020

Communicated by Hari Mohan Srivastava

Corresponding Author: Süleyman Çetinkaya, Faculty of Arts and Sciences, Department of Mathematics, P. O. Box 60, 41380 Kocaeli, Turkey | E-mail: suleyman.cetinkaya@kocaeli.edu.tr 2010 Mathematics Subject Classification. Primary 26A33; Secondary 34K37

(C) 2021 by University of Niš, Serbia | Creative Commons License: CC BY-NC-ND 
physical knowledge and physical properties of the nonlinear problem into account the exact solution of nonlinear FPDEs can be obtained. This knowledge gives us the idea about how numerical solutions of the nonlinear FPDEs can be constructed by the combination of Daftardar-Jafari method (DJM) and Laplace transform. In this study, Laplace Transform iterative method (LTIM) is extended to obtain solutions for time-space FPDEs. The LTIM method is employed to solve a variety of linear and nonlinear FPDEs. LTIM generally generates an accurate solution of FPDEs, which can be represented in terms of the fractional trigonometric functions or Mittag-Leffler functions. Moreover, it has been shown that semi-analytical methods with Laplace transform need fewer CPU time to compute the solutions of nonlinear fractional models, which are utilized in engineering and applied science. LTIM is a robust method to obtain solutions for distinct types of nonlinear and linear FPDEs. LTIM can decrease the time of calculation as well as error margin of the approximate solution.

\section{Preliminaries}

In this section, preliminaries, notations and features of the fractional calculus are given [1,2]. Riemann-Liouville time-fractional integral of a real valued function $u(x, t)$ is defined as

$$
I_{t}^{\alpha} u(x, t)=\frac{1}{\Gamma(\alpha)} \int_{0}^{t}(t-s)^{\alpha-1} u(x, s) d s
$$

where $\alpha>0$ denotes the order of the integral.

$\alpha^{t h}$ order the Liouville-Caputo time-fractional derivative operator of $u(x, t)$ is defined as

$$
\begin{aligned}
\frac{\partial^{\alpha} u(x, t)}{\partial t^{\alpha}} & =I_{t}^{m-\alpha}\left[\frac{\partial^{m} u(x, t)}{\partial t^{m}}\right] \\
& = \begin{cases}\frac{1}{\Gamma^{m-\alpha}-\alpha} \int_{0}^{t}(t-y)^{m-\alpha-1} \frac{\partial^{m} u(x, y)}{\partial y^{m}} d y, & m-1<\alpha<m, \\
\frac{\partial^{m}(x, t)}{\partial t^{m}}, & \alpha=m\end{cases}
\end{aligned}
$$

Mittag-Leffler function with two parameters is defined as

$$
E_{\alpha, \beta}(z)=\sum_{k=0}^{\infty} \frac{z^{k}}{\Gamma(\alpha k+\beta)}, \operatorname{Re}(\alpha)>0, z, \beta \in \mathbb{C}
$$

where $\alpha$ and $\beta$ are parameters.

The following set of functions has Laplace transformation

$$
\left\{f(t)\left|\exists M, \tau_{1}, \tau_{2}>0,\right| f(t) \mid<M e^{\frac{|t|}{\tau_{j}}}, \text { ift } \in(-1)^{j} \times[0, \infty)\right\}
$$


and it is defined as

$$
L[f(t)]=F(p)=\int_{0}^{\infty} e^{-p t} f(t) d t
$$

which has the following property

$$
L\left[t^{\alpha}\right]=\int_{0}^{\infty} e^{-p t} t^{\alpha} d t=\Gamma(\alpha+1)\left(\frac{1}{p}\right)^{\alpha+1}, \operatorname{Re}(\alpha)>0
$$

inverse Laplace inverse transform of $\left(\frac{1}{p}\right)^{n \alpha+1}$ is defined as

$$
L^{-1}\left[\left(\frac{1}{p}\right)^{n \alpha+1}\right]=\frac{t^{n \alpha}}{\Gamma(n \alpha+1)}, \operatorname{Re}(\alpha)>0
$$

where $n>0[2]$.

For $\alpha^{t h}$ order the Liouville-Caputo time-fractional derivative of $f(x, t)$, the Laplace transformation has the following form:

$$
L\left[\frac{\partial^{\alpha} f(x, t)}{\partial t^{\alpha}}\right]=p^{\alpha} L[f(x, t)]-\sum_{k=0}^{n-1}\left[p^{\alpha-k-1} \frac{\partial^{k} f(x, 0)}{\partial t^{k}}\right], n-1<\alpha \leqslant n, n \in \mathbb{N} .
$$

\section{Methodology}

In this section, we take the general time and space FPDE

$$
\frac{\partial^{\zeta} f}{\partial t^{\zeta}}=F\left(x, f, \frac{\partial^{\eta} f}{\partial x^{\eta}}, \ldots, \frac{\partial^{l \eta} f}{\partial x^{l \eta}}\right), j-1<\zeta \leqslant j, i-1<\eta \leqslant i, l, j, i \in \mathbb{N}
$$

along with the initial conditions

$$
\frac{\partial^{m} f(x, 0)}{\partial t^{m}}=h_{m}(x), k=0,1,2, \ldots, j-1,
$$

into account where $F\left(x, f, \frac{\partial^{\eta} f}{\partial x^{\eta}}, \ldots, \frac{\partial^{l \eta} f}{\partial x^{l \eta}}\right)$ could be linear or nonlinear and the function $f=f(x, t)$ is unknown. 
Applying the Laplace transform to both sides of Eq. (3.1) and rearranging leads to

$$
\begin{aligned}
L[f(x, t)] & =\sum_{m=0}^{j-1}\left[\left(\frac{1}{p}\right)^{m+1} \frac{\partial^{m} f(x, 0)}{\partial t^{m}}\right] \\
& +\left(\frac{1}{p}\right)^{\zeta+1} L\left[F\left(x, f, \frac{\partial^{\eta} f}{\partial x^{\eta}}, \ldots, \frac{\partial^{l \eta} f}{\partial x^{l \eta}}\right)\right] .
\end{aligned}
$$

Employing the inverse Laplace transform of Eq. (3.3), we obtain

$$
\begin{aligned}
f(x, t) & =L^{-1}\left[\sum_{m=0}^{j-1}\left[\left(\frac{1}{p}\right)^{m+1} \frac{\partial^{m} f(x, 0)}{\partial t^{m}}\right]\right] \\
& +L^{-1}\left[\left(\frac{1}{p}\right)^{\zeta+1} L\left[F\left(x, f, \frac{\partial^{\eta} f}{\partial x^{\eta}}, \ldots, \frac{\partial^{l \eta} f}{\partial x^{l \eta}}\right)\right]\right] .
\end{aligned}
$$

Equation (3.4) can be rearranged as

$$
f(x, t)=g(x, t)+G\left(x, f, \frac{\partial^{\eta} f}{\partial x^{\eta}}, \ldots, \frac{\partial^{l \eta} f}{\partial x^{l \eta}}\right)
$$

where

$$
\begin{aligned}
& g(x, t)=L^{-1}\left[\sum_{m=0}^{j-1}\left[\left(\frac{1}{p}\right)^{m+1} \frac{\partial^{m} f(x, 0)}{\partial t^{m}}\right]\right] \\
& G\left(x, f, \frac{\partial^{\eta} f}{\partial x^{\eta}}, \ldots, \frac{\partial^{l \eta} f}{\partial x^{l \eta}}\right)=L^{-1}\left[\left(\frac{1}{p}\right)^{\zeta+1} L\left[F\left(x, f, \frac{\partial^{\eta} f}{\partial x^{\eta}}, \ldots, \frac{\partial^{l \eta} f}{\partial x^{l \eta}}\right)\right]\right]
\end{aligned}
$$

Here $G$ is a nonlinear / linear operator and $g$ is known function. The solution of Eq. (3.5) can be obtained by the DJM introduced by Daftardar-Gejji and Jafari [16]. The solution is represented as an infinite series:

$$
f=\sum_{n=0}^{\infty} f_{n}
$$

where the terms $f_{n}$ are recursively computed. Decomposing the operator $G$ leads to 


$$
\begin{aligned}
& G\left(x, \sum_{n=0}^{\infty} f_{n}, \frac{\partial^{\eta}\left(\sum_{n=0}^{\infty} f_{n}\right)}{\partial x^{\eta}}, \ldots, \frac{\partial^{l \eta}\left(\sum_{n=0}^{\infty} f_{n}\right)}{\partial x^{l \eta}}\right) \\
& =G\left(x, f_{0}, \frac{\partial^{\eta} f_{0}}{\partial x^{\eta}}, \ldots, \frac{\partial^{l \eta} f_{0}}{\partial x^{l \eta}}\right) \\
& +\sum_{c=1}^{\infty}\left(G\left(x, \sum_{n=0}^{c} f_{n}, \frac{\partial^{\eta}\left(\sum_{n=0}^{c} f_{n}\right)}{\partial x^{\eta}}, \ldots, \frac{\partial^{l \eta}\left(\sum_{n=0}^{c} f_{n}\right)}{\partial x^{l \eta}}\right)\right) \\
& -\sum_{c=1}^{\infty}\left(G\left(x, \sum_{n=0}^{c-1} f_{n}, \frac{\partial^{\eta}\left(\sum_{n=0}^{c-1} f_{n}\right)}{\partial x^{\eta}}, \ldots, \frac{\partial^{l \eta}\left(\sum_{n=0}^{c-1} f_{n}\right)}{\partial x^{l \eta}}\right)\right)
\end{aligned}
$$

$$
\begin{aligned}
& L^{-1}\left[\left(\frac{1}{p}\right)^{\zeta+1} L\left[F\left(x, \sum_{n=0}^{\infty} f_{n}, \frac{\partial^{\eta}\left(\sum_{n=0}^{\infty} f_{n}\right)}{\partial x^{\eta}}, \ldots, \frac{\partial^{l \eta}\left(\sum_{n=0}^{\infty} f_{n}\right)}{\partial x^{l \eta}}\right)\right]\right] \\
& =L^{-1}\left[\left(\frac{1}{p}\right)^{\zeta+1} L\left[F\left(x, f_{0}, \frac{\partial^{\eta} f_{0}}{\partial x^{\eta}}, \ldots, \frac{\partial^{l \eta} f_{0}}{\partial x^{l \eta}}\right)\right]\right] \\
& +\sum_{c=1}^{\infty} L^{-1}\left[\left(\frac{1}{p}\right)^{\zeta+1} L\left[F\left(x, \sum_{n=0}^{c} f_{n}, \frac{\partial^{\eta}\left(\sum_{n=0}^{c} f_{n}\right)}{\partial x^{\eta}}, \ldots, \frac{\partial^{l \eta}\left(\sum_{n=0}^{c} f_{n}\right)}{\partial x^{l \eta}}\right)\right]\right] \\
& -\sum_{c=1}^{\infty} L^{-1}\left[\left(\frac{1}{p}\right)^{\zeta+1} L\left[F\left(x, \sum_{n=0}^{c-1} f_{n}, \frac{\partial^{\eta}\left(\sum_{n=0}^{c-1} f_{n}\right)}{\partial x^{\eta}}, \ldots, \frac{\partial^{l \eta}\left(\sum_{n=0}^{c-1} f_{n}\right)}{\partial x^{l \eta}}\right)\right]\right] .
\end{aligned}
$$

Using Eqs. (3.7), (3.9) in Eq. (3.5), we get

$$
\begin{aligned}
& \sum_{n=0}^{\infty} f_{n}=L^{-1}\left[\sum_{m=0}^{j-1}\left[\left(\frac{1}{p}\right)^{m+1} \frac{\partial^{m} f(x, 0)}{\partial t^{m}}\right]\right] \\
& +L^{-1}\left[\left(\frac{1}{p}\right)^{\zeta+1} L\left[F\left(x, f_{0}, \frac{\partial^{\eta} f_{0}}{\partial x^{\eta}}, \ldots, \frac{\partial^{l \eta} f_{0}}{\partial x^{l \eta}}\right)\right]\right] \\
& +\sum_{c=1}^{\infty} L^{-1}\left[\left(\frac{1}{p}\right)^{\zeta+1} L\left[F\left(x, \sum_{n=0}^{c} f_{n}, \frac{\partial^{\eta}\left(\sum_{n=0}^{c} f_{n}\right)}{\partial x^{\eta}}, \ldots, \frac{\partial^{l \eta}\left(\sum_{n=0}^{c} f_{n}\right)}{\partial x^{l \eta}}\right)\right]\right] \\
& -\sum_{c=1}^{\infty} L^{-1}\left[\left(\frac{1}{p}\right)^{\zeta+1} L\left[F\left(x, \sum_{n=0}^{c-1} f_{n}, \frac{\partial^{\eta}\left(\sum_{n=0}^{c-1} f_{n}\right)}{\partial x^{\eta}}, \ldots, \frac{\partial^{l \eta}\left(\sum_{n=0}^{c-1} f_{n}\right)}{\partial x^{l \eta}}\right)\right]\right] .
\end{aligned}
$$

The recurrence relation is defined by as follows: 


$$
\begin{aligned}
& f_{0}=L^{-1} {\left[\sum_{m=0}^{j-1}\left[\left(\frac{1}{p}\right)^{m+1} \frac{\partial^{m} f(x, 0)}{\partial t^{m}}\right]\right], } \\
& f_{1}=L^{-1}\left[\left(\frac{1}{p}\right)^{\zeta+1} L\left[F\left(x, f_{0}, \frac{\partial^{\eta} f_{0}}{\partial x^{\eta}}, \ldots, \frac{\partial^{l \eta} f_{0}}{\partial x^{l \eta}}\right)\right]\right], \\
& f_{r+1}=L^{-1}\left[\left(\frac{1}{p}\right)^{\zeta+1} L\left[F\left(x, \sum_{n=0}^{c} f_{n}, \frac{\partial^{\eta}\left(\sum_{n=0}^{c} f_{n}\right)}{\partial x^{\eta}}, \ldots, \frac{\partial^{l \eta}\left(\sum_{n=0}^{c} f_{n}\right)}{\partial x^{l \eta}}\right)\right]\right] \\
&-L^{-1}\left[\left(\frac{q}{p}\right)^{\zeta+1} L\left[F\left(x, \sum_{n=0}^{c-1} f_{n}, \frac{\partial^{\eta}\left(\sum_{n=0}^{c-1} f_{n}\right)}{\partial x^{\eta}}, \ldots, \frac{\partial^{l \eta}\left(\sum_{n=0}^{c-1} f_{n}\right)}{\partial x^{l \eta}}\right)\right]\right] .
\end{aligned}
$$

The $r$ - term truncated solution of Eqs. (3.1),(3.2) is constructed as $f \approx f_{0}+$ $f_{1}+\ldots+f_{r-1}$. We lead the reader to [17] for the convergence of DJM.

\section{Illustrative Example}

Let us consider time and space fractional equation below

$$
\frac{\partial^{\zeta} f}{\partial t^{\zeta}}=\left(\frac{\partial^{\eta} f}{\partial x^{\eta}}\right)^{2}-f\left(\frac{\partial^{\eta} f}{\partial x^{\eta}}\right), t>0, \zeta, \eta \in(0,1],
$$

along with the initial condition

$$
u(x, 0)=3+\frac{5}{2} E_{\eta}\left(x^{\eta}\right) .
$$

Let us apply the Laplace transform on both sides of (4.1).

$$
L\left[\frac{\partial^{\zeta} f}{\partial t^{\zeta}}\right]=L\left[\left(\frac{\partial^{\eta} f}{\partial x^{\eta}}\right)^{2}-f\left(\frac{\partial^{\eta} f}{\partial x^{\eta}}\right)\right]
$$

By means of the property (2.7), we obtain

$$
L[f(x, t)]=\left(\frac{1}{p}\right) f(x, 0)+\left(\frac{1}{p}\right)^{\zeta+1}\left(L\left[\left(\frac{\partial^{\eta} f}{\partial x^{\eta}}\right)^{2}-f\left(\frac{\partial^{\eta} f}{\partial x^{\eta}}\right)\right]\right) .
$$

Applying the inverse Laplace transform to both sides of Eq. (4.3) 


$$
f(x, t)=L^{-1}\left[\left(\frac{1}{p}\right) f(x, 0)\right]+L^{-1}\left[\left(\frac{1}{p}\right)^{\zeta+1}\left(L\left[\left(\frac{\partial^{\eta} f}{\partial x^{\eta}}\right)^{2}-f\left(\frac{\partial^{\eta} f}{\partial x^{\eta}}\right)\right]\right)\right]
$$

is obtained. Using the recurrence relation (3.10)

$$
\begin{aligned}
f_{0} & =L^{-1}\left[\left(\frac{1}{p}\right) f(x, 0)\right]=3+\frac{5}{2} E_{\eta}\left(x^{\eta}\right) \\
f_{1} & =L^{-1}\left[\left(\frac{1}{p}\right)^{\zeta+1}\left(L\left[\left(\frac{\partial^{\eta} f_{0}}{\partial x^{\eta}}\right)^{2}-f\left(\frac{\partial^{\eta} f_{0}}{\partial x^{\eta}}\right)\right]\right)\right]=-\frac{15 t^{\zeta} E_{\eta}\left(x^{\eta}\right)}{2 \Gamma(\zeta+1)} \\
f_{2} & =L^{-1}\left[\left(\frac{1}{p}\right)^{\zeta+1}\left(L\left[\left(\frac{\partial^{\eta}\left(f_{0}+f_{1}\right)}{\partial x^{\eta}}\right)^{2}-f\left(\frac{\partial^{\eta}\left(f_{0}+f_{1}\right)}{\partial x^{\eta}}\right)\right]\right)\right] \\
& -L^{-1}\left[\left(\frac{1}{p}\right)^{\zeta+1}\left(L\left[\left(\frac{\partial^{\eta} f_{0}}{\partial x^{\eta}}\right)^{2}-f\left(\frac{\partial^{\eta} f_{0}}{\partial x^{\eta}}\right)\right]\right)\right]=\frac{45 t^{2 \zeta} E_{\eta}\left(x^{\eta}\right)}{2 \Gamma(2 \zeta+1)} \\
f_{3} & =-\frac{135 t^{3 \zeta} E_{\eta}\left(x^{\eta}\right)}{2 \Gamma(3 \zeta+1)} \\
f_{4} & =-\frac{405 t^{4 \zeta} E_{\eta}\left(x^{\eta}\right)}{2 \Gamma(4 \zeta+1)}
\end{aligned}
$$

As a result, the series solution of the problem (4.1)-(4.2) is obtained by

$$
f(x, t)=f_{0}+f_{1}+f_{2}+f_{3}+\ldots=3+\left[\frac{5}{2} E_{\zeta}\left(-3 t^{\zeta}\right)\right] E_{\eta}\left(x^{\eta}\right),
$$

which gives the same solution as in [18].

\section{Conclusion}

LTIM is developed by taking the combination of DJM [16] and Laplace transform. This new approach is convenient for acquiring numerical solutions of time and space FPDEs. Its appicability is illustrated by an example in this study. As a result, the combination of DJM with Laplace transform provides a better and more effective approach than combination of Laplace transformation and homotopy, Sumudu or Adomian polynomials.

The results of this paper can be rewritten easily and trivially for any of the many rather inconsequential parameter and/or variable changes in the integral in Eq. (2.4) which defines the classical Laplace transform. 


\section{Acknowledgement}

The authors gratefully thank to Prof. Dr. H.M. Srivastava for the constructive comments and recommendations which definitely help to improve the readability and quality of the paper.

\section{R E F E R E N C E S}

1. A. A. Kilbas, H. M. Srivastava and J. J. Trujillo: Theory and applications of fractional differential equations, Elsevier, Amsterdam, 2006.

2. I. Podlubny: Fractional Differential Equations: An Introduction to Fractional Derivatives, Fractional Differential Equations, to Methods of Their Solution and Some of Their Applications. Academic Press, New York, 1998.

3. H. M. SRIVAstava and Z. Tomovski: Fractional Calculus with an Integral Operator Containing a Generalized Mittag-Leffler Function in the Kernel. Applied Mathematics and Computation 2111 (2019), 198-210.

4. Z. Tomovski, R. Hilfer and H.M. Srivastava: Fractional and Operational Calculus with Generalized Fractional Derivative Operators and Mittag-Leffler Type Functions, Integral Transforms and Special Functions 2111 (2010), 797-814.

5. S. Cetinkaya, A. Demir and H. Kodal Sevindir: The analytic solution of initial boundary value problem including time-fractional diffusion equation. Facta Universitatis Ser. Math. Inform 351 (2020), 243-252. DOI Number: https://doi.org/10.22190/FUMI2001243C.

6. S. Cetinkaya, A. Demir and H. Kodal Sevindir: The analytic solution of sequential space-time fractional diffusion equation including periodic boundary conditions. Journal of Mathematical Analysis 111 (2020), 17-26.

7. S. CetinkayA and A. DemiR: The Analytic Solution of Time-Space Fractional Diffusion Equation via New Inner Product with Weighted Function. Communications in Mathematics and Applications, 104 (2019), 865-873, DOI:http://dx.doi.org/10.26713

8. S. Cetinkaya, A. Demir and H. Kodal Sevindir: The Analytic Solution of Initial Periodic Boundary Value Problem Including Sequential Time Fractional Diffusion Equation. Communications in Mathematics and Applications 111 (2020), 173-179, DOI: $10.26713 /$ cma.v11i1.1293

9. S. Cetinkaya and A. Demir: Sequential space fractional diffusion equation's solutions via new inner product. Asian-European Journal of Mathematics (2021), https://doi.org/10.1142/S1793557121501217

10. S. CetinkayA and A. DemiR: Time Fractional Equation with Non-homogenous Dirichlet Boundary Conditions. Sakarya University Journal of Science 246 (2020), 1185-1190.

11. S. Cetinkaya and A. DemiR: Time Fractional Diffusion Equation with Periodic Boundary Conditions. Konuralp Journal of Mathematics 82 (2020), 337-342.

12. Y. F. Luchko and H. M. SRIVAstava: The Exact Solution of Certain Differential Equations of Fractional Order by Using Operational Calculus. Computers and Mathematics with Applications 298 (1995), 73-85. 
13. K. V. Zhukovsky and H. M. SRivastava: Analytical solutions for heat diffusion beyond Fourier law. Applied Mathematics and Computation 293 (2017), 423-437.

14. L. Mahto, S. Abbas, M. Hafayed and H. M. Srivastava: Approximate Controllability of Sub-Diffusion Equation with Impulsive Condition. Mathematics $\mathbf{7} 190$ (2019), 1-16.

15. X. J. Yang, H. M. Srivastava, D. F. M. Torres and A. Debbouche: General Fractional-order Anomalous Diffusion with Non-singuler Power-Law Kernel. Thermal Science 211 (2017), 1-9.

16. V. DAFtardar-GejJI and H. JAFARI: An iterative method for solving nonlinear functional equations. J. Math. Anal. Appl. 3162 (2006), 753-763.

17. S. Bhalekar and V. Daftardar-GejJi: Convergence of the new iterative method. International Journal of Differential Equations (2011), DOI:10.1155/2011/989065

18. S. Choudhary and V. Daftardar-GejJi: Invariant subspace method: a tool for solving fractional partial differential equations. Fract. Calc. Appl. Anal. 202 (2017), 477-493. 